\title{
Frequencies of SF3B1, NOTCH1, MYD88, BIRC3 and IGHV mutations and TP53 disruptions in Chinese with chronic Iymphocytic leukemia: disparities with Europeans
}

\author{
Yi Xia ${ }^{1}$, Lei Fan ${ }^{1}$, Li Wang ${ }^{1}$, Robert Peter Gale ${ }^{2}$, Man Wang ${ }^{1}$, Tian Tian ${ }^{1}$, Wei Wu ${ }^{1}$, \\ Liang $\mathbf{Y u}^{3}$, Yao-Yu Chen ${ }^{1}$, Wei $\mathbf{X u}^{1}$ and Jian-Yong $\mathbf{L i}^{1}$ \\ ${ }^{1}$ Department of Hematology, The First Affiliated Hospital of Nanjing Medical University, Jiangsu Province Hospital, Nanjing, \\ China \\ 2 Haematology Research Centre, Division of Experimental Medicine, Department of Medicine, Imperial College London, \\ London, United Kingdom \\ ${ }^{3}$ Department of Hematology, Huai'an First People's Hospital, Nanjing Medical University, Huai'an, Jiangsu Province, China \\ Correspondence to: Wei Xu, email: xuwei10000@medmail.com.cn
}

Jian-Yong Li, email: lijianyonglm@medmail.com

Keywords: SF3B1, NOTCH1, MYD88, mutation, chronic lymphocytic leukemia

Received: September 01,2014 Accepted: December 29,2014Ｐublished: December 31, 2014

This is an open-access article distributed under the terms of the Creative Commons Attribution License, which permits unrestricted use, distribution, and reproduction in any medium, provided the original author and source are credited.

\section{ABSTRACT}

We studied 307 consecutive Chinese with chronic lymphocytic leukemia (CLL) in diverse disease-stages before and after diverse therapies for mutations in several CLL-related genes. Mutation frequencies were SF3B1, 5\%, NOTCH1, 8\%, MYD88, 8\%, BIRC3, 2\%, TP53, 15\% and IGHV, 60\%. Several of these frequencies differ from those reported in persons of predominately European descent with CLL. Biological and clinical associations were detected including SF3B1 and NOTCH1 mutations with un-mutated IGHV, MYD88 mutations with mutated IGHV, SF3B1 mutations with fludarabineresistant CLL and NOTCH1 mutation with advanced Binet disease stage and with +12 . The NOTCH1 correlation with briefer survival was confirmed in multivariate analyses but the SF3B1 correlation was confounded by concurrent mutations in TP53 and germline IGHV. We show differences in incidence and prognostic impact of mutations in Chinese and CLL compared with persons of predominately European descent with CLL. These data may give insights into the etiology and biology of CLL and suggests different risk stratification models may be needed for different CLL populations.

\section{INTRODUCTION}

Biology and clinical course of chronic lymphocytic leukemia (CLL) are heterogeneous. An unfavorable prognosis is associated with certain cytogenetic and molecular abnormalities such as del(11q22-q23), TP53 disruption and un-mutated immunoglobulin heavychain variable region $(I G H V)$ state [1-3]. Mutations in RNA-splicing and processing, genes involved in Notch signaling, inflammatory pathway genes and genes in the Wnt pathway are also associated with briefer survival of persons with CLL [4].

Mutations in SF3B1, NOTCH1, MYD88 and BIRC3 are relatively rare in persons of predominately European descent with CLL with frequencies of $5-18 \%, 8-12 \%$, $\sim 3 \%$, and $\sim 2 \%$ [4-10]. However, mutations of these genes in a sub-clone of CLL cells undetectable by conventional sequencing may become detectable as CLL progresses. It is also likely some new mutations are acquired as CLL progresses with or without therapy [11]. We studied frequencies and prognostic associations of mutations of SF3B1, NOTCH1, MYD88, BIRC3, TP53 and IGHV and cytogenetic abnormalities in 307 Chinese with CLL.

\section{RESULTS}

Subjects

Characteristics of the 307 subjects are summarized in Table 1. Median age was 61 years (range, 16- 92 
Table 1: Clinical characteristics

\begin{tabular}{|c|c|}
\hline & N (\%) \\
\hline Sex $(\mathrm{n}=307)$ & $204(66)$ \\
\hline Male & $103(34)$ \\
\hline Female & $164(53)$ \\
\hline Age $(\mathrm{n}=307)$ & $143(47)$ \\
\hline$\geq 60$ & $129(43)$ \\
\hline$<60$ & $70(24)$ \\
\hline Binet $(\mathrm{n}=297)$ & $98(33)$ \\
\hline A & \\
\hline B & $166(54)$ \\
\hline C & $88(29)$ \\
\hline $\begin{array}{c}\text { Disease States } \\
(\mathrm{n}=307)\end{array}$ & $25(8)$ \\
\hline At diagnosis & $28(9)$ \\
\hline Progressive & \\
\hline Relapsed & \\
\hline Refractory & \\
\hline
\end{tabular}

years) with a male/female ratio of $2: 1$. Median follow-up is 35 months (range, 1-267 months),

\section{SF3B1, NOTCH1, MYD88, BIRC3 and TP53 mutations}

307 subjects were studied for SF3B1 mutations. 15 $(5 \%)$ had a $S F 3 B 1$ mutation. All mutations were missense with p.K700E the most frequent $(\mathrm{N}=10) .24$ of $295(8 \%)$ of subjects analyzed had NOTCH1 mutations of whom 17 had c.7544-7545delCT. Other mutations were nonsense $(\mathrm{N}=3)$ or out-of-frame $(\mathrm{N}=4)$. Two mutations, p.L2049fs*1 and p.Q2404X, were not reported by COSMIC or other studies. Mutations in exons 3-5 of MYD88 were detected in $23(8 \%)$ of 295 subjects analyzed. The most frequent mutation was p.L265P $(\mathrm{N}=15)$. p.V271F is a new mutation. 5 of $238(2 \%)$ had a BIRC3 mutation including 4 out-of-frame and 1 in-frame. 47 (15\%) of 307 subjects analyzed had TP53 mutations. Most mutations were in the DNA-binding domain ( $\mathrm{N}=45 ; 94 \%)$.

Subjects with $S F 3 B 1$ mutation were more likely to have concomitant TP53 mutation (5 of 15 vs. 42 of 292; $P=0.047)$. Also, MYD88 mutations were exclusive of NOTCH1 and SF3B1 mutations. Detailed data are presented in Supplement Table 1.

\section{IGHV Mutations}

In 118 of 299 (40\%) subjects $I G H V$ was germline. The most frequently used $I G H V$ genes were $I G H V 4-34$ $(\mathrm{N}=38 ; 13 \%), I G H V 3-23(\mathrm{~N}=33 ; 11 \%), I G H V 3-7(\mathrm{~N}=30$, $10 \%), I G H V 4-39(\mathrm{~N}=21 ; 7 \%), I G H V 4-59 \mathrm{~N}=15 ; 5 \%)$, $I G H V 1-69(\mathrm{n}=15 ; 5 \%)$ and $I G H V 3-21(\mathrm{~N}=9 ; 3 \%)$.

\section{Clinical correlations}

NOTCH1 mutations were detected more frequently in subjects with advanced Binet stages (stage-A, 4 of 126; stage-B: 6 of 66 ; stage-C, 14 of $94 ; P=0.008$ ). TP53 mutations were also more common in subjects with advanced Binet stage (stage-A, 11 of 129; stage-B, 12 of 70 ; stage-C, 23 of 98; $P=0.008$ ). We found no significant correlation between Binet stage and $S F 3 B 1$ or $M Y D 88$ mutations (Table 3 ) but power to detect an association is limited because of the low frequency of mutations. MYD88 mutations were less frequent in subjects in whom CD38 was $\geq 30 \%$ ( 1 of $67 v s, 22$ of $223 ; P=0.026$ ). NOTCH1 mutations were associated with CD38 $\geq 30 \%$ but this difference was not significant (9 of 67 vs. 15 of $223 ; P=0.081$ ). We found no correlation between $S F 3 B 1$ or TP53 mutations and CD38 $\geq 30 \%$. There was also no correlation between any of the mutations we studied and ZAP70 $\geq 20 \%$. BIRC3 mutations were excluded from this

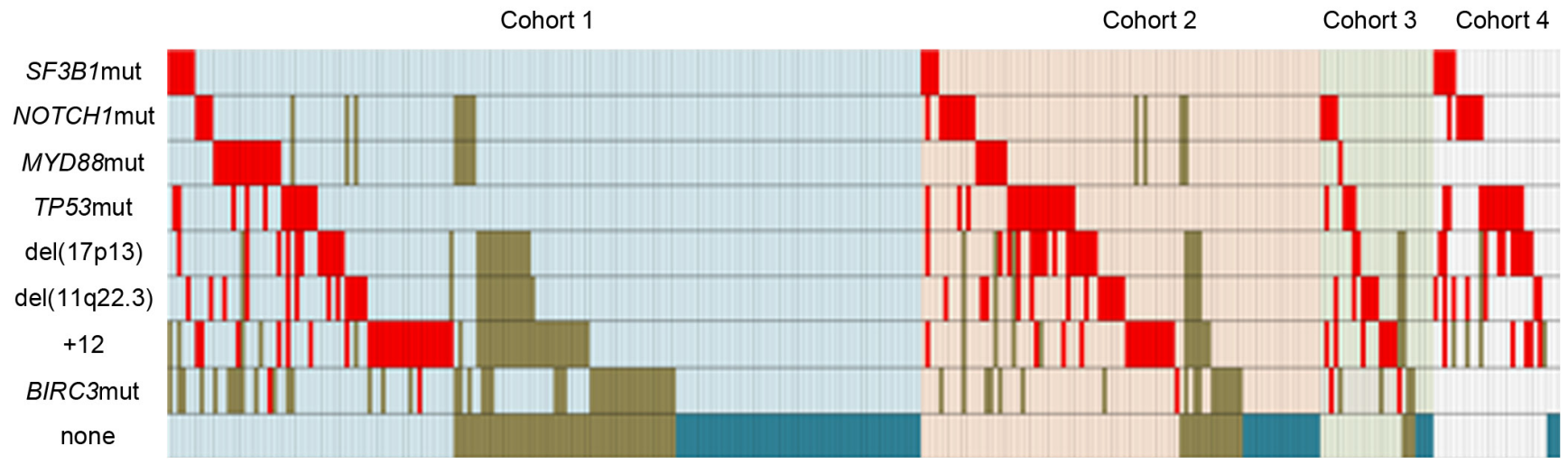

Figure 1: Relationship between TP53, SF3B1, NOTCH1 and MYD88 mutations, and cytogenetic abnormalities in 307 subjects with data. Cases which presented with a cytogenetic aberration or a mutation are colored in red. Cohort1: Newly-diagnosed; cohort 2: Progressive; no therapy; cohort 3:Relapsed CLL after therapy; and cohort 4: Refractory, therapy-resistant. Subjects are highlighted in light blue, pink, light green and light purple, respectively. Brown means not analyzed. 


\begin{tabular}{|}
\hline Table 2: Mutation frequency by disease state. \\
\hline & Diagnosis & Progressive & Relapsed & Refractory \\
\hline$S F 3 B 1$ & $6 / 166(4 \%)$ & $4 / 88(5 \%)$ & $0 / 25$ & $5 / 28(18 \%)$ \\
\hline NOTCH1 & $4 / 158(3 \%)$ & $9 / 84(11 \%)$ & $4 / 25(16 \%)$ & $7 / 28(25 \%)$ \\
\hline$M Y D 88$ & $15 / 158(10 \%)$ & $7 / 84(8 \%)$ & $1 / 25(4 \%)$ & $0 / 28$ \\
\hline$B I R C 3$ & $2 / 119(2 \%)$ & $1 / 71(1.4 \%)$ & $2 / 21(10 \%)$ & $0 / 27$ \\
\hline$T P 53$ & $13 / 166(8 \%)$ & $18 / 84(21 \%)$ & $4 / 25(16 \%)$ & $12 / 28(43 \%)$ \\
\hline
\end{tabular}

\begin{tabular}{|l|l|l|l|l|l|l|l|l|l|}
\hline \multicolumn{2}{|l|}{ Table 3: Associations between subject variables and mutations } \\
\hline & & SF3B1mut & $\boldsymbol{P}$ & NOTCH1mut & $\boldsymbol{P}$ & MYD88mut & $\boldsymbol{P}$ & TP53mut & $\boldsymbol{P}$ \\
\hline $\mathrm{N}$ & 307 & $15 / 307(5 \%)$ & - & $24 / 295(8 \%)$ & - & $19 / 229(8 \%)$ & - & $47 / 307(15 \%)$ & - \\
\hline Female & $103(34 \%)$ & $2 / 103(2 \%)$ & 0.089 & $7 / 101(7 \%)$ & 0.585 & $6 / 101(6 \%)$ & 0.391 & $21 / 103(20 \%)$ & 0.093 \\
\hline Male & $204(66 \%)$ & $13 / 204(6 \%)$ & & $17 / 194(9 \%)$ & & $17 / 194(9 \%)$ & & $26 / 204(13 \%)$ & \\
\hline Age & 61 & $61( \pm 12)$ & 0.911 & $64( \pm 11)$ & 0.212 & $62( \pm 8)$ & 0.631 & $61( \pm 13)$ & 0.890 \\
\hline Binet A & $129(43 \%)$ & $5 / 129(4 \%)$ & 0.305 & $4 / 126(3 \%)$ & $\mathbf{0 . 0 0 8}$ & $9 / 126(7 \%)$ & 0.680 & $11 / 129(9 \%)$ & $\mathbf{0 . 0 0 8}$ \\
\hline Binet B & $70(24 \%)$ & $6 / 70(9 \%)$ & & $6 / 66(9 \%)$ & & $4 / 66(6 \%)$ & & $12 / 70(17 \%)$ & \\
\hline Binet C & $98(33 \%)$ & $4 / 98(4 \%)$ & & $14 / 94(15 \%)$ & & $9 / 94(10 \%)$ & & $23 / 98(24 \%)$ & \\
\hline CD38 $\geq 30 \%$ & $71 / 302(24 \%)$ & $5 / 71(7 \%)$ & 0.356 & $9 / 67(13 \%)$ & 0.081 & $1 / 67(2 \%)$ & $\mathbf{0 . 0 2 6}$ & $10 / 71(14 \%)$ & 0.677 \\
\hline CD38<30\% & $231 / 302(76 \%)$ & $10 / 231(4 \%)$ & & $15 / 223(7 \%)$ & & $22 / 223(10 \%)$ & & $36 / 231(16 \%)$ & \\
\hline ZAP70 $20 \%$ & $110 / 272(40 \%)$ & $7 / 110(6 \%)$ & 0.613 & $11 / 106(10 \%)$ & 0.246 & $6 / 106(6 \%)$ & 0.523 & $17 / 110(16 \%)$ & 0.774 \\
\hline ZAP70<20\% & $162 / 272(60 \%)$ & $8 / 162(5 \%)$ & & $10 / 156(6 \%)$ & & $12 / 156(8 \%)$ & & $23 / 162(14 \%)$ & \\
\hline IGHV M & $181 / 299(61 \%)$ & $5 / 181(3 \%)$ & $\mathbf{0 . 0 2 7}$ & $2 / 172(1 \%)$, & $<\mathbf{0 . 0 0 1}$ & $21 / 172(12 \%)$ & $\mathbf{0 . 0 0 1}$ & $19 / 181(11 \%)$ & $\mathbf{0 . 0 0 1}$ \\
\hline IGHV UM & $118 / 299(40 \%)$ & $10 / 118(9 \%)$ & & $21 / 115(18 \%)$ & & $2 / 115(2 \%)$ & & $28 / 118(24 \%)$ & \\
\hline
\end{tabular}
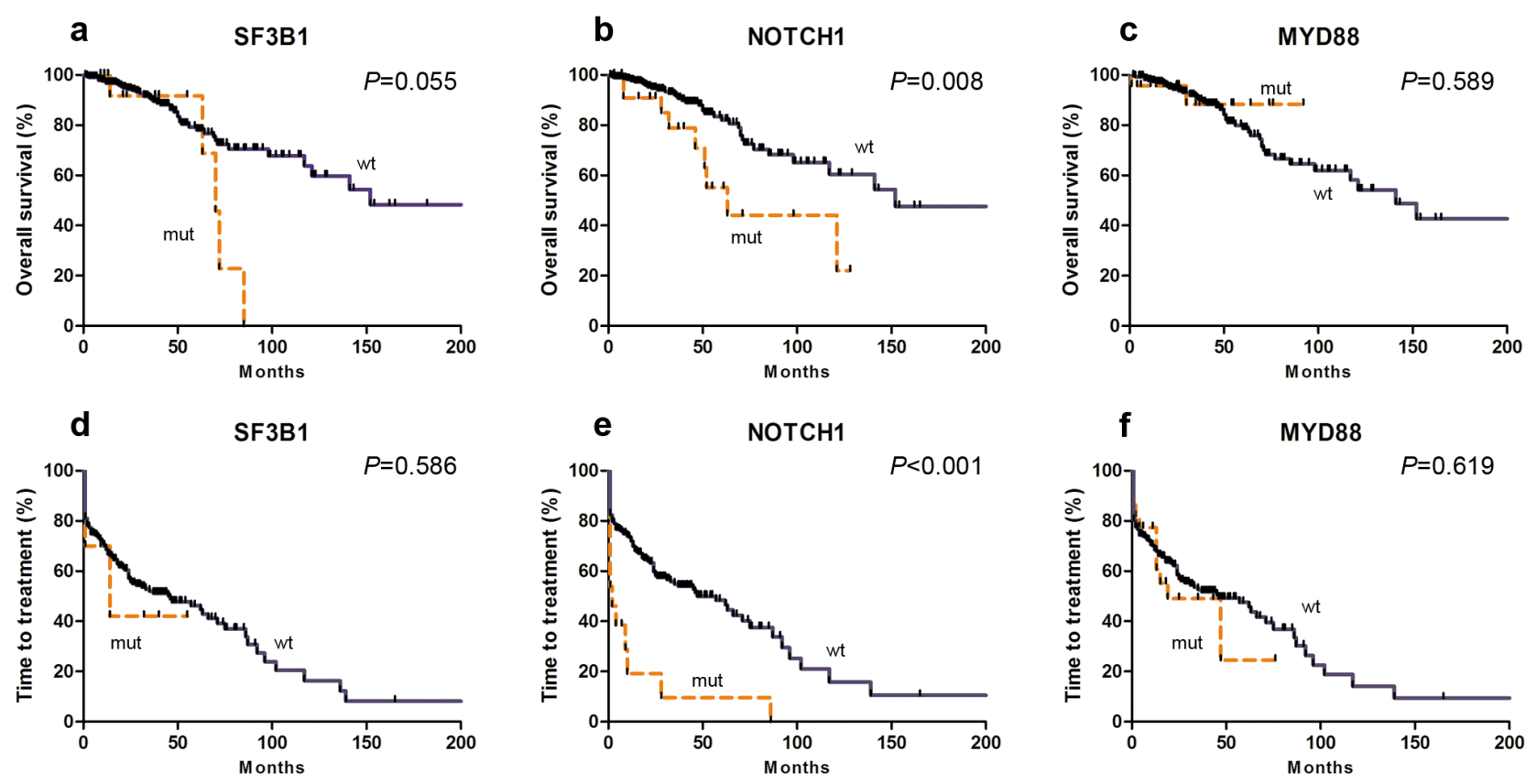

Figure2: Kaplan-Meier curves of survival (a-c) and time-to-treatment (TTT) (d-f) for SF3B1 (a and d), NOTCH1 (b and e) and MYD88 mutations (c and f). 


\begin{tabular}{|c|c|c|c|c|c|c|}
\hline \multirow[b]{2}{*}{ Variable } & \multicolumn{3}{|c|}{ Time-to-treatment } & \multicolumn{3}{|c|}{ Survival } \\
\hline & $\mathrm{N}$ & Median (mo) & P-value & $\mathrm{N}$ & Median (mo) & P-value \\
\hline TP53 disruption & 234 & & 0.003 & 285 & & $<0.001$ \\
\hline Yes & 44 & 12 & & 64 & 71 & \\
\hline No & 190 & 62 & & 221 & NR & \\
\hline $\operatorname{del}(11 \mathrm{q} 22.3)$ & 232 & & 0.061 & 281 & & 0.030 \\
\hline Yes & 28 & 18 & & 42 & 77 & \\
\hline No & 204 & 47 & & 239 & 152 & \\
\hline+12 & 211 & & 0.393 & 257 & & 0.366 \\
\hline Yes & 44 & 37 & & 59 & 117 & \\
\hline No & 167 & 66 & & 198 & 152 & \\
\hline$S F 3 B 1$ & 254 & & 0.586 & 307 & & 0.055 \\
\hline Mutated & 10 & 14 & & 15 & 72 & \\
\hline Wild-type & 244 & 46 & & 292 & 152 & \\
\hline NOTCH1 & 242 & & $<0.001$ & 295 & & 0.008 \\
\hline Mutated & 13 & 2 & & 24 & 63 & \\
\hline Wild-type & 229 & 57 & & 271 & 152 & \\
\hline MYD88 & 242 & & 0.619 & 295 & & 0.589 \\
\hline Mutated & 22 & 19 & & 23 & NR & \\
\hline Wild-type & 220 & 46 & & 272 & 141 & \\
\hline
\end{tabular}

analysis because they were so rare (Table 3 ).

SF3B1, NOTCH1 and TP53 mutations were significantly correlated with germline $I G H V(S F 3 B 1,10$ of $118 v s .5$ of $181 ; P=0.027$; NOTCH1, 21 of $115 v s .2$ of 170; $P<0.001$; TP53, 28 of 118 vs. 19 of 181: $P=0.002$ ). In contrast, MYD 88 mutations were more common in subjects with mutated IGHV ( 2 of 115 vs. 21 of 172; $P=0.001$; Table 3). NOTCH1 mutations were especially common in subjects using IGHV4-39 (4 of 19 vs. 19 of 268; $P=0.03$ ) and $I G H V 1-69$ (6 of 15 vs. 17 of 255; $P<0.001)$. SF3B1 mutations were correlated with IGHV459 (4 of 15 vs. 11 of $284 ; P=0.004$ ).

\section{Association with cytogenetic abnormalities}

Cytogenetic abnormalities were common in our subjects including +12 in 59 of 257 (23\%), del(17p13) in 42 of $281(15 \%)$ and del(11q22.3) in 40 of $283(14 \%)$. As expected, NOTCH1 mutations were significantly associated with +12 ( 9 of 56 vs. 12 of $192 ; P=0.020$ ) and TP53 mutations with del(17p13) (23 of 40 vs. 22 of 243; $P<0.001)$. Among the 64 subjects with TP53 abnormalities, 17 had only del(17p13), 23 had TP53 mutation and $\operatorname{del}(17 \mathrm{p} 13)$ and 22 only TP53 mutation (2 missed del(17p13) data, but got TP53 mutation). Surprisingly, there was no correlation between $S F 3 B 1$ mutations and del(11q22.3) (4 of 42 vs. 11 of 239; $P=0.191)$. MYD 88 mutations did not correlate with cytogenetic abnormalities (Supplement Figure 1). Detailed distribution of mutations and cytogenetic lesions are shown in Figure 1.

\section{Prognostic relevance of mutations and cytogenetic abnormalities}

We divided subjects into 4 prognostic cohorts: (1) newly-diagnosed; (2) progressive; no therapy; (3) relapsed CLL after therapy; and (4) refractory, fludarabine-resistant. Frequency of gene mutations in each cohort is shown in Table 2. Mutation rate of $S F 3 B 1$ was significantly higher in subjects in cohort $4(P=0.007)$. NOTCH1 was less frequently mutated in subjects in cohort $1(P<0.001)$ but similar in cohorts 2-4 $(P>0.05)$. TP53 mutation was less frequent in subjects in cohort $1(P=0.004)$ and more common in subjects in cohort $4(P=0.033)$. MYD 88 mutation was more common in subjects in cohort 1 and 2 $(P<0.001)$. There was no significant association of BIRC3 mutation with any cohort.

Prognostic impact of the abnormalities we studied is shown in Figure 2. Survival was significantly briefer in subjects with NOTCH1 mutation (median, 63 vs. 153 months; $P=0.008)$, del(11q22.3) (median, 77 vs. 152 months; $P=0.030$ ) and TP53 disruptions (median, 71 months vs. NR, $P<0.001)$. MYD88 mutations had no significant impact on survival (median, NR vs. 142 months; $P=0.590$ ). However, $S F 3 B 1$ mutations had only a borderline impact on survival (median, 70 vs. 152 months; $P=0.055$ ) even when 6 subjects with concurrent TP53 mutations were included. The frequency of BIRC3 mutations in BIRC3 was too low for statistical comparisons (Supplement Table 2 ).

We divided the TP53 mutation cohort into 2 cohorts based on $I G H V$ rearrangement. 30 of 64 subjects with 
TP53 disruption and mutated $I G H V$ had longer survival compared with those with TP53 disruption and germline $I G H V$ (median, 141 vs. 60 months; $P=0.001$ ). Survival of the favorable cohort was not significantly different from subjects without TP53 disruptions (median, 141 months vs. NR; $P=0.308$; Figure $3 \mathrm{a}$ ). There was also no significant survival difference with only TP53 mutation, only del(17p13) or both $(P=0.474)$.

Next, we compared whether subjects with TP53 disruptions and other unfavorable abnormalities including del(11q22.3) or mutations in NOTCH1 and/or SF3B1 mutations had shorter survival compared with those with TP53 disruptions only. There was no significant difference (median, 70 vs. 71 months; $P=0.540$; Figure 3c). Multiple unfavorable abnormalities were more common in subjects with refractory, fludarabine-resistant disease (cohort 4; 6 of 143 vs. 14 of 127; $P=0.033$; Supplement Table 3).

The impact of each mutation on TTT was studied in previously untreated subjects (cohort 1 and 2; Table 4). Subjects with NOTCH1 mutations and/or TP53 disruptions had briefer TTT than subjects without these abnormalities (median, 2 vs. 57 months; $P<0.001$ and 12 vs. 62 months; $P=0.003)$. Subjects with TP53 disruptions and mutated $I G H V$ had similar TTT as subjects without TP53 disruptions (median: 46 vs. 62 months; $P=0.423$ ). There was no significant correlation between mutations in SF3B1 or MYD88 mutation and TTT (median, $14 \mathrm{vs.}$ 46 months; $P=0.586$; median, 19 vs. 46 months; $P=0.619$; Figure 2 and $3 b$ ).

In multivariate analyses variables independentlycorrelated with TTT included germline $\operatorname{IGHV}(\mathrm{HR}=2.30$, 95\% CI: $1.51-3.49 ; \quad P<0.001), \quad$ TP53 disruptions $(\mathrm{HR}=1.85,95 \% \mathrm{CI}: 1.20-2.83 ; P=0.005)$ and NOTCH1 mutation $(\mathrm{HR}=2.17,95 \% \mathrm{CI}: 1.11-4.23 ; P=0.024)$

\section{DISCUSSION}

We provide data of frequencies of mutations in SF3B1, NOTCH1, MYD88, BIRC3 in Chinese with CLL along with data on previously described prognostic variables including TP53 disruptions, IGHV mutation and cytogenetic abnormalities. One striking difference was the frequency, biological features and prognostic impact of SF3B1 mutations in Chinese with CLL. The frequency we detected is considerably lower than reported in persons of predominately European descent (Table 2) [4, 7, 8, 1214]. This low rate might be accounted for by the relatively low sensitivity of the Sanger sequencing we used. For example, Jeromin et al. reported about 10\% SF3B1 mutations occurred at a mutation load $\leq 10 \%$, a level detectable only with next generation sequencing (NGS) and would have been missed by us [13]. However, this is unlikely to explain the disparate frequencies we observed. We found $S F 3 B 1$ mutation frequency was significantly higher in subjects with advanced, fludarabine-resistant CLL consistent with the notion detection of these mutations result from expansion of a sub-clone of CLL cells and are acquired during disease progression $[15,16]$.

We also found discrepancies regarding to $I G H V$ gene use by $S F 3 B 1$ mutated cases between our subjects and persons of predominately European descent. Strefford et al. reported a much higher frequency of $S F 3 B 1$ mutations in stereotyped $I G H V 3-21$ [17]. A recent study of 1160 untreated persons of predominately European descent with CLL reported frequent $S F 3 B 1$ mutations in subjects with IGHV3-21 and IGHV1-69 gene use [13]. Many of our subjects had germline $I G H V$. In contrast, $S F 3 B 1$ mutations were not correlated with $I G H V 1-69$ or $I G H V 3-21$ but rather with IGHV4-59. This low frequency of IGHV1-69 use in Chinese with CLL is previously reported [18, 19]. Only 9 of 299 subjects had IGHV321 use and only 15 used $I G H V 1-69$. Furthermore, only one IGHV1-69 user had SF3B1 p.K700E mutation. The reason for the disparities between Chinese and persons of European descent with CLL are unknown and could relate to the low incidence of $S F 3 B 1$ mutations and IGHV1-69 and IGHV3-21 use in Chinese with CLL. It is also possible different antigenic stimuli operate in diverse geographies [20]. Interestingly, $S F 3 B 1$ mutation was not associated with advanced Binet stage, CD38 $\geq 30 \%$ or $\operatorname{del}(11 \mathrm{q} 22.3$ ) but was correlated with TP53 mutation. These data suggest
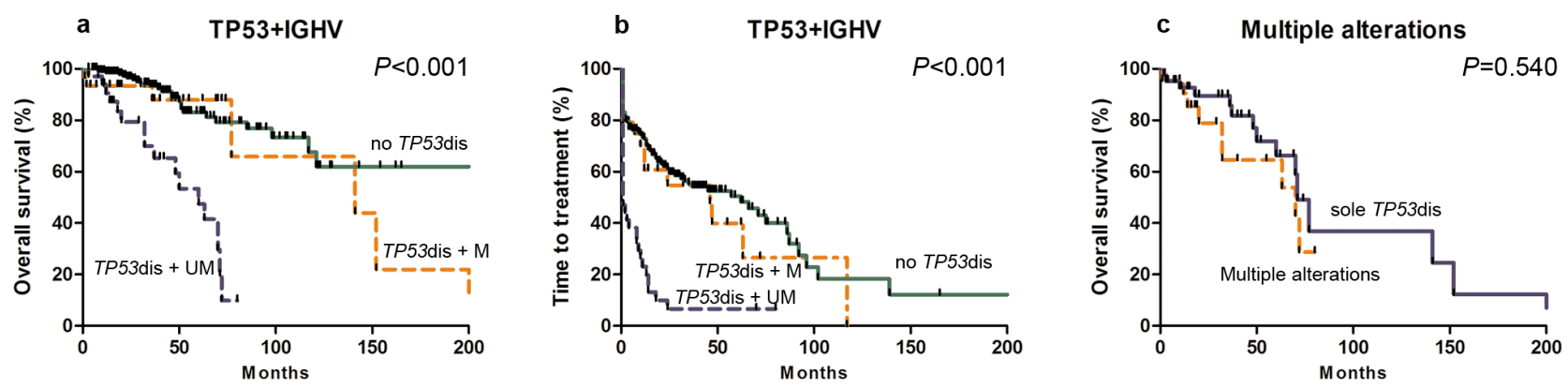

Figure3: Kaplan-Meier curves of survival (a) and TTT (b) for TP53 disruption cases with different IGHV gene mutation states. Comparison of survival subjects with multiple unfavorable alterations including TP53 vs. those with TP53 only mutation is shown in (c). 
distinct biological features of $S F 3 B 1$ mutated cases of CLL in Chinese may differ from those in persons of predominately European decent.

Incidence of NOTCH1 mutations in our cohort is relatively low at diagnosis but increased with disease progression. These data are compatible with a sub-clone of CLL cells below the sensitivity of Sanger sequencing followed by clonal expansion during disease course, acquisition of additional mutations spontaneously for as a consequence of therapy or both $[11,16]$. Mutation detection methods with higher sensitivity, such as allele specific PCR, should be able to distinguish these possibilities which are not mutually-exclusive [21]. The hot spot c.7544-7545delCT deletion accounted for about $70 \%$ of our mutated cases. Notably, two novel nonsense mutations were found suggesting the possibility of novel NOTCH1 mutation sites. Significant associations were observed between $\mathrm{NOTCH} 1$ mutations and advanced Binet stage, germline $I G H V$ and +12 . IGHV4-39 and IGHV1-69 were frequently used in subjects with $\mathrm{NOTCH1}$ mutations. +12 and $\mathrm{NOTCH} 1$ mutations are reported to characterize IGHV4-39 CLL belonging to subset 8 and with higher risk of transformation to diffuse large B-cell lymphoma (DLBCL; Richter syndrome) [22, 23]. We observed only 1 case of transformation, possibly because of brief followup.

Another interesting finding is the high frequency of MYD88 mutation in our cohort [12-14]. MYD88 mutation is common in subjects with mutated $I G H V$ and rare in the CD38 $\geq 30 \%$ cohort. Moreover, almost all MYD 88 mutations occurred in untreated subjects consistent with the hypothesis MYD88 mutation occurs early in CLL development [11]. In addition, we found no significant correlation between BIRC3 mutation in fludarabineresistant patients as previously reported [10].

We show NOTCH1 and TP53 disruptions are unfavorable factors for survival and TTT consistent with prior studies in persons of predominately European descent $[5,6,8,11-14,16]$. In contrast, the prognostic impact of $S F 3 B 1$ mutations is modest, if any. The unfavorable impact of $S F 3 B 1$ mutation on survival was largely dependent on concurrent mutations in TP53 and germline $I G H V$. Our data contradict other studies in persons of predominately European descent in whom $S F 3 B 1$ mutation is a strong independent predictor of brief survival $[4,7,8,10,11-16]$. We had, of course, little power to test the impact of SF3B1 mutation alone on survival because of the low frequency so our conclusion should be viewed cautiously.

We found high rate of subjects with TP53 mutations in persons without del(17p13). These subjects had a poor prognosis similar to those with both abnormalities similar to our prior report [24]. However, we also found a cohort of subjects with TP53 disruptions had stable disease. Subjects with TP53 disruptions and germline IGHV had the worst prognosis whereas those with TP53 disruptions and mutated $I G H V$ gene had longer TTT and survival similar to subjects without TP53 abnormality. Others report similar data emphasizing the combination of $I G H V$ rearrangement and TP53 disruptions might be a better predictor of prognosis than either variable alone [14, 25].

Recently, Greipp et al. reported persons with CLL with del(17p13) and del(11q22.3) had briefer survival compared with persons with del(17p13). These persons were termed double hit CLL [26]. Based on this report we compared outcomes of persons with TP53 disruptions and other unfavorable abnormalities including del(11q22.3) or mutations in NOTCH1 and/or SF3B1 mutations with those with TP53 mutation only. We found no significant difference in outcomes. There are several possible explanations for this disparity including different populations, different $N O T C H 1$ and $S F 3 B 1$ mutations and different cut-off values for FISH in the 2 studies. Nevertheless, our data support the concept many unfavorable genetic abnormalities are more common in advanced CLL.

In multivariate analyses, NOTCH1 mutation, germline $I G H V$ and TP53 disruptions were independently correlated with TTT. Detection of NOTCH1 mutations helped us reclassify 13 subjects with no other unfavorable risk factors. Patients with NOTCH1 and/or SF3B1 mutations were considered intermediate to high risk in other reports [12-14]. In our cohort NOTCH1 mutation had the greatest risk of requiring therapy which would also indicate high-risk disease. In contrast, SF3B1 mutations had little or no prognostic impact and were included in the good-risk cohort. These disparities highlight the need for different risk classifications for different populations with CLL.

In conclusion, we studied frequency and prognostic impact of cytogenetic and molecular abnormalities in a large series of Chinese with CLL and compared these data with data from persons of predominately European descent with CLL. Our study highlights important similarities but also which may offer important clues to the etiology and biology of CLL.

\section{METHODS}

\section{Subjects}

The study cohort was a single-center consecutive series of 307 Chinese with CLL. Subjects were diagnosed from November, 1991 to April, 2014. Subjects provided informed consent according to institutional guidelines. Diagnosis of CLL was based on International Workshop on CLL-National Cancer Institute (IWCLL-NCI) criteria [27]. 


\section{Analyses of SF3B1, NOTCH1, MYD88 mutations and TP53 disruptions}

Genomic DNA was isolated from mononuclear cells using the QIAamp DNA Blood Kits (Qiagen, Düsseldorf, Germany) according to the manufacturer's recommendation. Direct Sanger sequencing was performed for exon 14-16 of SF3B1, PEST domain of NOTCH1, exon 3-5 of MYD88, exon 6-9 of BIRC3 and exon 4-9 of TP53. Primers are listed in Supplement Table 4.

\section{Cytogenetics}

Fluorescence in situ hybridization (FISH) analysis was performed on most subjects to detect of del(11q22.3) $(\mathrm{n}=281)$, del(17p13) $(\mathrm{n}=283)$ and trisomy $12(\mathrm{n}=257)$. The following fluorescent-labeled probes were used: LSI ATM (11q22.3), LSI p53 (17p13) and CEP12 (centromere 12). Probes were purchased from Vysis, Downers Grove, IL, USA. FISH was performed as described [24]. Cut-off levels for positivity were $7.7 \%, 5.2 \%$, and $3.0 \%$ for del(11q22.3), del(17p13) and +12 . Although not all subjects with TP53 mutation were analyzed for del(17p13), we refer the cohort with TP53 mutation or del(17p13) with no TP53 mutation testing as TP53 disruptions.

\section{Immunophenotyping and IGHV mutation analyses}

Immunophenotyping of CD38 and ZAP-70 expression and $I G H V$ sequencing were performed as described [28]. Positive cut-off values were $30 \%$ and $20 \%$. Germline $I G H V$ was defined as $\geq 98 \%$ germline homology.

\section{Statistical analyses}

Survival was calculated as time from diagnosis until death or last follow-up. Time-to-treatment (TTT) was calculated as time from diagnosis until first treatment. Calculations were performed using SPSS (version 19.0) software (IBM Corporation, Armonk, NY, USA). Categorical variables were compared using $\chi^{2}$ test and continuous variables using Student t-test. Survival curves were constructed by Kaplan-Meier method and log-rank test was used for significant associations. Multivariate analysis was done by Cox proportional hazard regression. Multivariate analysis of TTT was done. However, there were too few events to analyze survival. $P$-values were two-sided; $P<0.05$ was considered significant .

\section{ACKNOWLEDGEMENTS}

This study was supported by National Natural Science Foundation of China (Grant Nos. 30871104, 30971296, 81000216, 81100352, 81170485, 81170486, $81170488,81200360,81300408,81370657)$, Natural Science Foundation of Jiangsu Province (Grant No. BK2012484, BK20141028), Key Projects of Health Department of Jiangsu Province (Grant No. K201108), Jiangsu Province's Medical Elite Program (Grant No. RC2011169), National Public Health Grand Research Foundation (Grant No.201202017), Program for Development of Innovative Research Teams in the First Affiliated Hospital of Nanjing Medical University, and Project of National Key Clinical Specialty, National Science \& Technology Pillar Program (Grant No.2014BAI09B12), and Project funded by Jiangsu Provincial Special Program of Medical Science (Grant No.BL2014086). RPG acknowledges support from the NIHR Biomedical Research Centre funding scheme.

\section{REFERENCES}

1. Hamblin TJ, Davis Z, Gardiner A, Oscier DG and Stevenson FK. Unmutated $\operatorname{Ig} \mathrm{V}(\mathrm{H})$ genes are associated with a more aggressive form of chronic lymphocytic leukemia. Blood. 1999; 94(6):1848-1854.

2. Dohner H, Stilgenbauer S, Benner A, Leupolt E, Krober A, Bullinger L, Dohner K, Bentz M and Lichter P. Genomic aberrations and survival in chronic lymphocytic leukemia. The New England journal of medicine. 2000; 343(26):19101916.

3. Dicker F, Herholz H, Schnittger S, Nakao A, Patten N, Wu L, Kern W, Haferlach T and Haferlach C. The detection of TP53 mutations in chronic lymphocytic leukemia independently predicts rapid disease progression and is highly correlated with a complex aberrant karyotype. Leukemia. 2009; 23(1):117-124.

4. Wang L, Lawrence MS, Wan Y, Stojanov P, Sougnez C, Stevenson K, Werner L, Sivachenko A, DeLuca DS, Zhang L, Zhang W, Vartanov AR, Fernandes SM, Goldstein NR, Folco EG, Cibulskis K, et al. SF3B1 and other novel cancer genes in chronic lymphocytic leukemia. The New England journal of medicine. 2011; 365(26):2497-2506.

5. Puente XS, Pinyol M, Quesada V, Conde L, Ordonez GR, Villamor N, Escaramis G, Jares P, Bea S, Gonzalez-Diaz M, Bassaganyas L, Baumann T, Juan M, Lopez-Guerra M, Colomer D, Tubio JM, et al. Whole-genome sequencing identifies recurrent mutations in chronic lymphocytic leukaemia. Nature. 2011; 475(7354):101-105.

6. Fabbri G, Rasi S, Rossi D, Trifonov V, Khiabanian H, Ma J, Grunn A, Fangazio M, Capello D, Monti S, Cresta S, Gargiulo E, Forconi F, Guarini A, Arcaini L, Paulli M, et al. Analysis of the chronic lymphocytic leukemia coding genome: role of NOTCH1 mutational activation. The 
Journal of experimental medicine. 2011; 208(7):1389-1401.

7. Quesada V, Conde L, Villamor N, Ordonez GR, Jares P, Bassaganyas L, Ramsay AJ, Bea S, Pinyol M, MartinezTrillos A, Lopez-Guerra M, Colomer D, Navarro A, Baumann T, Aymerich M, Rozman M, et al. Exome sequencing identifies recurrent mutations of the splicing factor SF3B1 gene in chronic lymphocytic leukemia. Nature genetics. 2012; 44(1):47-52.

8. Stilgenbauer S, Schnaiter A, Paschka P, Zenz T, Rossi M, Dohner K, Buhler A, Bottcher S, Ritgen M, Kneba M, Winkler D, Tausch E, Hoth P, Edelmann J, Mertens D, Bullinger L, et al. Gene mutations and treatment outcome in chronic lymphocytic leukemia: results from the CLL8 trial. Blood. 2014.

9. Foa R, Del Giudice I, Guarini A, Rossi D and Gaidano G. Clinical implications of the molecular genetics of chronic lymphocytic leukemia. Haematologica. 2013; 98(5):675685.

10. Rossi D, Fangazio M, Rasi S, Vaisitti T, Monti S, Cresta S, Chiaretti S, Del Giudice I, Fabbri G, Bruscaggin A, Spina V, Deambrogi C, Marinelli M, Fama R, Greco M, Daniele $\mathrm{G}$, et al. Disruption of BIRC3 associates with fludarabine chemorefractoriness in TP53 wild-type chronic lymphocytic leukemia. Blood. 2012; 119(12):2854-2862.

11. Landau DA, Carter SL, Stojanov P, McKenna A, Stevenson K, Lawrence MS, Sougnez C, Stewart C, Sivachenko A, Wang L, Wan Y, Zhang W, Shukla SA, Vartanov A, Fernandes SM, Saksena G, et al. Evolution and impact of subclonal mutations in chronic lymphocytic leukemia. Cell. 2013; 152(4):714-726.

12. Rossi D, Rasi S, Spina V, Bruscaggin A, Monti S, Ciardullo C, Deambrogi C, Khiabanian H, Serra R, Bertoni F, Forconi F, Laurenti L, Marasca R, Dal-Bo M, Rossi FM, Bulian $\mathrm{P}$, et al. Integrated mutational and cytogenetic analysis identifies new prognostic subgroups in chronic lymphocytic leukemia. Blood. 2013; 121(8):1403-1412.

13. Jeromin S, Weissmann S, Haferlach C, Dicker F, Bayer K, Grossmann V, Alpermann T, Roller A, Kohlmann A, Haferlach T, Kern W and Schnittger S. SF3B1 mutations correlated to cytogenetics and mutations in NOTCH1, FBXW7, MYD88, XPO1 and TP53 in 1160 untreated CLL patients. Leukemia. 2014; 28(1):108-117.

14. Cortese D, Sutton LA, Cahill N, Smedby KE, Geisler C, Gunnarsson R, Juliusson G, Mansouri L and Rosenquist R. On the way towards a 'CLL prognostic index': focus on TP53, BIRC3, SF3B1, NOTCH1 and MYD88 in a population-based cohort. Leukemia. 2014; 28(3):710-713.

15. Rossi D, Bruscaggin A, Spina V, Rasi S, Khiabanian H, Messina M, Fangazio M, Vaisitti T, Monti S, Chiaretti S, Guarini A, Del Giudice I, Cerri M, Cresta S, Deambrogi C, Gargiulo E, et al. Mutations of the SF3B1 splicing factor in chronic lymphocytic leukemia: association with progression and fludarabine-refractoriness. Blood. 2011; 118(26):69046908.

16. Schnaiter A, Paschka P, Rossi M, Zenz T, Buhler A,
Winkler D, Cazzola M, Dohner K, Edelmann J, Mertens D, Kless S, Mack S, Busch R, Hallek M, Dohner H and Stilgenbauer S. NOTCH1, SF3B1, and TP53 mutations in fludarabine-refractory CLL patients treated with alemtuzumab: results from the CLL2H trial of the GCLLSG. Blood. 2013; 122(7):1266-1270.

17. Strefford JC, Sutton LA, Baliakas P, Agathangelidis A, Malcikova J, Plevova K, Scarfo L, Davis Z, Stalika E, Cortese D, Cahill N, Pedersen LB, di Celle PF, Tzenou T, Geisler C, Panagiotidis P, et al. Distinct patterns of novel gene mutations in poor-prognostic stereotyped subsets of chronic lymphocytic leukemia: the case of SF3B1 and subset \#2. Leukemia. 2013; 27(11):2196-2199.

18. Chen L, Zhang Y, Zheng W, Wu Y, Qiao C, Fan L, Xu $\mathrm{W}$ and Li J. Distinctive IgVH gene segments usage and mutation status in Chinese patients with chronic lymphocytic leukemia. Leukemia research. 2008; 32(10):1491-1498.

19. Agathangelidis A, Darzentas N, Hadzidimitriou A, Brochet X, Murray F, Yan XJ, Davis Z, van Gastel-Mol EJ, Tresoldi C, Chu CC, Cahill N, Giudicelli V, Tichy B, Pedersen LB, Foroni L, Bonello L, et al. Stereotyped B-cell receptors in one-third of chronic lymphocytic leukemia: a molecular classification with implications for targeted therapies. Blood. 2012; 119(19):4467-4475.

20. Rosen A, Murray F, Evaldsson C and Rosenquist R. Antigens in chronic lymphocytic leukemia--implications for cell origin and leukemogenesis. Seminars in cancer biology. 2010; 20(6):400-409.

21. Bo MD, Del Principe MI, Pozzo F, Ragusa D, Bulian P, Rossi D, Capelli G, Rossi FM, Niscola P, Buccisano F, Bomben R, Zucchetto A, Maurillo L, de Fabritiis P, Amadori S, Gaidano G, et al. NOTCH1 mutations identify a chronic lymphocytic leukemia patient subset with worse prognosis in the setting of a rituximab-based induction and consolidation treatment. Annals of hematology. 2014.

22. Rossi D, Spina V, Bomben R, Rasi S, Dal-Bo M, Bruscaggin A, Rossi FM, Monti S, Degan M, Ciardullo C, Serra R, Zucchetto A, Nomdedeu J, Bulian P, Grossi A, Zaja F, et al. Association between molecular lesions and specific B-cell receptor subsets in chronic lymphocytic leukemia. Blood. 2013; 121(24):4902-4905.

23. Rossi D, Rasi S, Spina V, Fangazio M, Monti S, Greco M, Ciardullo C, Fama R, Cresta S, Bruscaggin A, Laurenti L, Martini M, Musto P, Forconi F, Marasca R, Larocca LM, et al. Different impact of NOTCH1 and SF3B1 mutations on the risk of chronic lymphocytic leukemia transformation to Richter syndrome. British journal of haematology. 2012; 158(3):426-429.

24. Dong HJ, Zhou LT, Zhu DX, Wang DM, Fang C, Zhu HY, Zhuang Y, Miao KR, Xu W and Li JY. The prognostic significance of TP53 mutations in Chinese patients with chronic lymphocytic leukemia is independent of del(17p13). Annals of hematology. 2011; 90(6):709-717.

25. Best OG, Gardiner AC, Davis ZA, Tracy I, Ibbotson RE, 
Majid A, Dyer MJ and Oscier DG. A subset of Binet stage A CLL patients with TP53 abnormalities and mutated IGHV genes have stable disease. Leukemia. 2009; 23(1):212-214.

26. Greipp PT, Smoley SA, Viswanatha DS, Frederick LS, Rabe KG, Sharma RG, Slager SL, Van Dyke DL, Shanafelt TD, Tschumper RC and Zent CS. Patients with chronic lymphocytic leukaemia and clonal deletion of both $17 \mathrm{p} 13.1$ and $11 \mathrm{q} 22.3$ have a very poor prognosis. British journal of haematology. 2013; 163(3):326-333

27. Hallek M, Cheson BD, Catovsky D, Caligaris-Cappio F, Dighiero G, Dohner H, Hillmen P, Keating MJ, Montserrat E, Rai KR and Kipps TJ. Guidelines for the diagnosis and treatment of chronic lymphocytic leukemia: a report from the International Workshop on Chronic Lymphocytic Leukemia updating the National Cancer Institute-Working Group 1996 guidelines. Blood. 2008; 111(12):5446-5456.

28. Xu W, Li JY, Wu YJ, Yu H, Shen QD, Li L, Fan L and Qiu HX. Prognostic significance of ATM and TP53 deletions in Chinese patients with chronic lymphocytic leukemia. Leukemia research. 2008; 32(7):1071-1077. 